\title{
RECENT EXHIBITIONS
}

Gutenberg began, five hundred years ago, both the art and the business of printing. As a commercial enterprise, printing has been remarkably successful during the five centuries of its history, while the less commercial aspects of printing have had, to say the least, their ups and downs. In spite of that, an exhibition for the months of September and October proved that examples of fine printing could be found in every decade from 1460 to the present. The exhibition served several other purposes as well. It showed that the earliest period of printing was also the most artistic, that books printed before I 600 were often produced by craftsmen who learned their trade from master printers in direct descent from the fountain heads of Gutenberg, Fust, and Schoeffer. It showed that even during the "dark ages" of printing, individual printers like Bodoni, the Elzevirs, and the Foulis brothers and men who had a knowledge of typographical tradition, like John Fell, kept the tradition of fine printing alive. It showed finally that "progress" was no blessing for the graphic arts, that the period of the beginning of the steam press was a low ebb in fine printing, and that only by disregarding economics could private presses like the Lee Priory Press and William Morris's Kelmscott Press produce works of typographical distinction. The illustration shown on the opposite page stated this major theme of the exhibition, that the "progress" of printing was like a squirrel in a cage, and that true improvement of typographical standards was more likely to arise from a study of tradition than from any technological improvements. The books exhibited were the following, all from the Rutgers University Library:

$\begin{array}{lll}\text { 1460 } & \text { Gutenberg } & \text { Page from the Catholicon } \\ \text { I479 } & \text { Filippo de Pietro } & \text { Justinus, Epitoma } \\ \text { I485 } & \text { Zarotus } & \text { Horace } \\ \text { I497 } & \text { Schonsperger } & \text { Schedel, Liber Chronicarum } \\ \text { I50 I } & \text { André Bocard } & \text { Lyndewood, Provinciale } \\ \text { I515 } & \text { Aldus Manutius } & \text { Lactantius } \\ \text { I524 } & \text { Johann Froben } & \text { Aesop } \\ \text { I532 } & \text { Hans Schotten } & \text { Brunfels, Kreüterbuch } \\ \text { I544 } & \text { Robert Estienne } & \text { Horace } \\ \text { I558 } & \text { Steelsius } & \text { Cumiranus } \\ \text { I561 } & \text { John Kyngston } & \text { Chaucer } \\ \text { I579 } & \text { Arnold Birckmann } & \text { Cicero } \\ \text { I581 } & \text { Christopher Plantin } & \text { Guicciardini }\end{array}$




\begin{tabular}{|c|c|c|}
\hline 594 & Henri Estienne & de Tyrranis Heracleae Ponticae \\
\hline 609 & Lodewijk Elzevir & Batavia Illustrata \\
\hline 611 & Robert Barker & Holy Bible \\
\hline 625 & Elzevir & Camden, Annals of Elizabeth \\
\hline 1630 & Willem Blaeu & Dares and Dictys \\
\hline 1642 & Elzevir & Heinsius, Orations \\
\hline 658 & Elzevir & Rufus, Historia Alexandri Magni \\
\hline 669 & Elzevir & Variorum Justinus \\
\hline 673 & Henry Hall & Dugard, Marriage of Cousins German \\
\hline 688 & Oxford University Press & Broadside (facs.) \\
\hline I694 & Benjamin Harris (Boston) & Makemere, Answer to George Keith \\
\hline I 704 & van $\operatorname{der} \mathrm{Aa}$ & Arrianus, Expeditionis Alexandri \\
\hline 711 & Plantin Press & Missale Romanum \\
\hline 1720 & Lintot & Pope, Iliad \\
\hline 732 & Franklin & Poor Richard's Almanack \\
\hline I 744 & Franklin & Cicero, Cato Major \\
\hline 759 & Baskerville & Milton, Paradise Lost \\
\hline 63 & Joachim Ibarra & Duhamel de Monceau, Memorias \\
\hline $77^{8}$ & $\begin{array}{l}\text { William and Andrew } \\
\text { Foulis }\end{array}$ & \\
\hline 780 & Didot & Combles, $L^{\prime} E$ cole $d u$ \\
\hline I 799 & Bodoni & Parini, $O d i$ \\
\hline 1808 & Bodoni & Iliad \\
\hline I8I4 & Lee Priory Press & Breton, Longing of a Blessed Heart \\
\hline 1823 & Nichols & . of Queen Elizabeth \\
\hline 1833 & Chiswick Press & Ritson, Ancient Popular Poetry \\
\hline 848 & Gift Book & Young Ladies Offering \\
\hline 185 & $\begin{array}{l}\text { New Jersey Historical } \\
\text { Society }\end{array}$ & Lewis Morris Papers \\
\hline 1860 & Chiswick Press & Liber Vagatorum \\
\hline 1873 & Chiswick Press & Diary of Lady Willoughby \\
\hline 1889 & Ballantyne Press & Dictionary of Slang, Jargon, and Cant \\
\hline 1891 & Kelmscott Press & Morris, Poems by the Way \\
\hline$O I$ & Elston Press & Tale of Gamelyn \\
\hline 913 & Doves Press & Prospice \\
\hline 1924 & Hogarth Press & Henry James at Work \\
\hline 37 & Oriole Press & Prospectus \\
\hline 43 & Bruce Rogers & Paragraphs on Printing. \\
\hline 50 & Overbrook Press & "Progress" in the Graphic Arts \\
\hline
\end{tabular}

Other exhibitions included a group of modern children's books, on loan from the American Institute of Graphic Arts, the customary Rutgersensia material in September, and a group of broadsides, ballots, and manuscript material pertaining to New Jersey elections in October. 


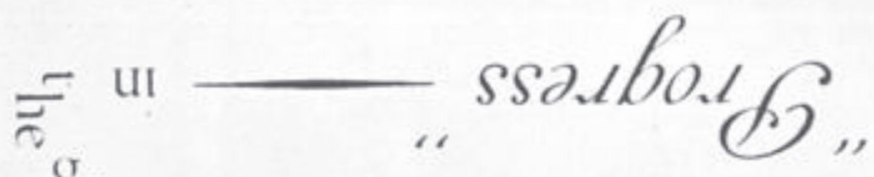
$\mathrm{graph}_{\mathrm{ic}}$ arts
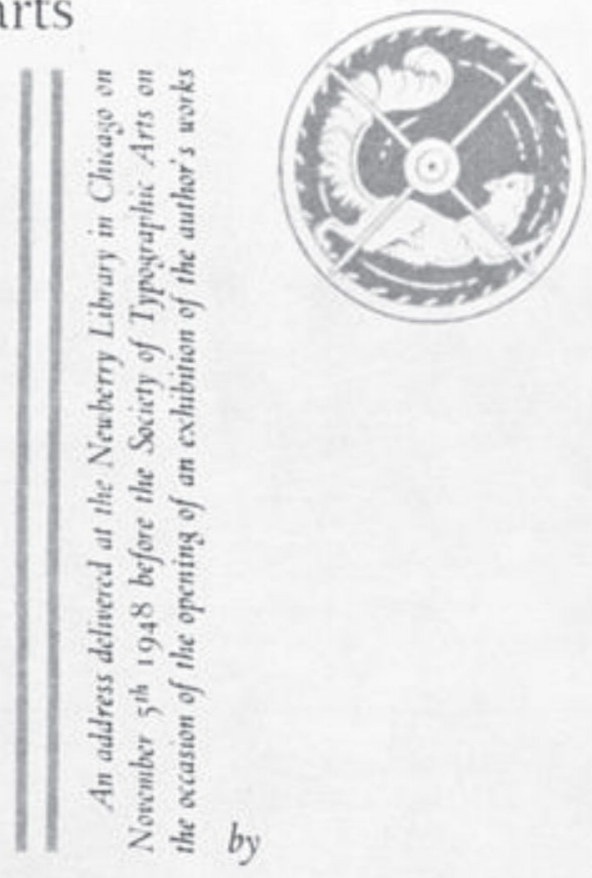

ดNจาสาว $W$ I

Printed in privacy

THE OVERBROOK PRESS

1950

STAMFORD, CONNECTICUT

Title page for "Progress" in the Graphic Arts, designed by T. M. Cleland 